Horizons philosophiques

\title{
La philosophie de Spinoza, l'éthique et la vérité
}

\section{Pierre Gendron}

Volume 5, numéro 1, automne 1994

Esthétiques et sociétés

URI : https://id.erudit.org/iderudit/800970ar

DOI : https://doi.org/10.7202/800970ar

Aller au sommaire du numéro

\section{Éditeur(s)}

Collège Édouard-Montpetit

\section{ISSN}

1181-9227 (imprimé)

1920-2954 (numérique)

Découvrir la revue

\section{Citer cet article}

Gendron, P. (1994). La philosophie de Spinoza, l'éthique et la vérité. Horizons philosophiques, 5(1), 116-132. https://doi.org/10.7202/800970ar d'utilisation que vous pouvez consulter en ligne.

https://apropos.erudit.org/fr/usagers/politique-dutilisation/ 


\section{LA PHILOSOPHIE DE SPINOZA, L'ÉTHIQUE ET LA VÉRITÉ}

"Ne pouvant fortifier la justice, on a justifié la force, afin que le juste et le fort fussent ensemble et que la paix fût, qui est le souverain bien."

Pascal

L'éthique est-elle un stratagème? La «persévérance dans l'être" de chaque individu est sans doute l'expression qui caractérise le mieux l'éthique chez Spinoza. Or l'individu n'est pas seul; et dans la mesure où sa conduite prend une signification dans la communication humaine et par l'emploi du langage, moyen de communication, il ne peut éluder la question de la vérité. Le but du présent article est d'élucider la nature du rapport entre la justice, qui permet à chacun de persévérer pleinement dans son être, et la vérité. La démarche proposée consistera en une interprétation des textes et de la pensée de Spinoza concernant des notions entre lesquelles le lien n'est pas a priori évident, telles la fermeté, la générosité et le salut par la connaissance. Une précaution s'avère toutefois nécessaire avant de commencer; il sera essentiel de ne jamais perdre de vue un principe de lecture, qui tient en une formule et qui commande tout le reste : veritas norma sui et falsi est ${ }^{1}$.

La «force d'âme» est la forme que prend la persévérance dans l'être sous la conduite de la raison. Or, Spinoza divise la force d'âme; il ne la réduit pas à la fermeté animositas : "Je

1. Le texte latin des œuvres de Spinoza auquel se réfère le présent essai est celui de l'édition de Carl Gebhardt, Spinoza Opera, Heidelberg, Carl Winters, 1925. Le tome II de cette édition contient l'ouvrage en cinq parties intitulé par Spinoza Ethica ordine geometrico demonstrata; le tome III contient les vingt chapitres du Tractatus theologico-politicus. La traduction française utilisée est celle de Charles Appuhn. "Qui a une idée vraie, affirme Spinoza, sait en même temps qu'il a une idée vraie et ne peut douter de la vérité de sa connaissancen" (Éthique, II, 43, Oeuvres, t. III, Paris, Garnier-Flammarion, 1965, p. 117). Et certes, ajoute-t-il, "comme la lumière se fait connaître elle-même et fait connaître les ténèbres, la vérité est norme d'ellemême et du faux - norma sui et falsìn (ibid., scolie, p. 118). 
ramène à la force d'âme, dit-il, les affections se rapportant à l'âme en tant qu'elle connaît, et je divise la force d'âme en fermeté et générosité. Par fermetéj'entends un désir par lequel un individu s'efforce à se conserver en vertu du seul commandement de la raison. Par générositéj'entends un désir par lequel un individu s'efforce en vertu du seul commandement de la raison à assister les autres hommes et à établir entre eux et lui un lien d'amitié. Je rapporte donc à la fermeté ces actions qui ont pour but l'utilité de l'agent seulement, et à la générosité celles qui ont aussi pour but l'utilité d'autrui "'. Par là, une opposition est mise en œuvre entre la fermeté, d'une part, qui consiste à "se conserver» et la générosité, d'autre part, qui consiste à "assister les autres hommes". Un ordre estégalement établi entre les termes de cette opposition: par rapport aux actions qui se rapportent à la fermeté, les actions qui se rapportent à la générosité sont simplement celles qui ont aussi pour but l'utilité d'autrui. Mais cet ordre est-il le seul ordre intelligible?

La philosophie se trouve au centre de cette articulation de la fermeté et de la générosité; car agir en vertu du seul commandement de la raison, c'est agir en philosophe (la raison fondant le lien d'amitié dont parle Spinoza). Or, agir en philosophe, être philosophe pour Spinoza n'est le fait que du petit nombre. C'est pourquoi il admettra, dans le chapitre XV du Tractatus theologico-politicus, une voie de salutparl'obéissance en vertu du témoignage de l'Écriture. À la distinction entre la fermeté et la générosité, viendra donc s'ajouter la séparation de la foi et de la philosophie; et avec elle la possibilité d'un certain déplacement de l'articulation.

«Par fermeté, dit donc Spinoza, j'entends un désir par lequel un individu s'efforce conatur à se conserver en vertu du seul commandement de la raison". Se situant en deçà de cette raison, la démarche proposée ici consistera d'abord à se demander : quelle est la "stratégie» du désir auquel Spinoza 
rapporte les «actions qui ont pour but l'utilité de l'agent seulement"?

La conduite "éthique», selon Spinoza, a pour principe l'effort pour se conserver qui est «la première et unique origine de la vertu ${ }^{3}$ ". Cet effort de persévérance dans l'être, en tant qu'il a conscience de soi, est ce que Spinoza appelle le désir, qui est "l'essence même de l'homme" 4 , puisque de manière générale "l'essence actuelle" de chaque chose rei actualem essentiam est l'effort conatus de persévérer dans son être. Quelle est donc, encore une fois, la "stratégie» ${ }^{5}$ de ce désir par lequel l'individu s'efforce à se conserver?

D'abord "l'homme pense", affirme Spinoza6; la stratégie de son désir est élaborée dans la réflexion. L'homme pense en tant qu'il a certaines idées ${ }^{7}$; il peut élaborer des stratégies en vertu du principe suivant lequel "l'ordre et la connexion des idées sont les mêmes que l'ordre et la connexion des choses" $"$. Or, ses stratégies seront bonnes dans la mesure où les idées qu'il formera seront adéquates, c'est-à-dire vraies considérées en elles-mêmes ${ }^{9}$. Et puisque (selon Spinoza) les hommes ne naissent pas libres, le problème est de savoir comment ils arrivent à former la première fois des idées adéquates. S'ils y arrivent, c'est gagné; car les idées qui suivent d'idées adéquates sont nécessairement adéquates ${ }^{10}$.

3. Ibid., IV, 22, corollaire, p. 240.

4. Ibid., III, 9, scolie, p. 145.

5. L'emploi du terme de stratégie, bien qu'il ne soit pas de Spinoza, s'autorise malgré tout d'un passage du Traité théologico-politique, chap. III, où l'auteur explique clairement que : "Pour vivre en sécurité et éviter les attaques des autres hommes et aussi des bêtes, le gouvernement de la vie humaine et la vigilance sont d'un grand secours. Et la raison etl'expérience ont enseigné que le plus sûr moyen d'y atteindre est la formation d'une société ayant des lois bien établies, l'occupation d'une certaine région du monde et la réunion en un même corps social des forces de tous" (Spinoza, Oeuvres, t. II, Paris, Garnier-Flammarion, 1965, p. 72).

6. Éthique, II, axiome 2, op. cit., p. 70.

7. Ibid., définition 3, p. 69.

8. Ibid., proposition 7, p. 75.

9. Ibid., définition 4, p. 70.

10. Ibid., proposition 40, p. 112. 
Pour Spinoza, chaque corps est caractérisé par un ensemble de propriétés qui sont comme des parties dans un tout, lequel est défini par un certain rapport. Les corps, en effet, sont dits se convenir ou s'accorder dans la mesure où ils ont certaines de leurs propriétés en commun. L'idée d'une telle intersection des rapports est ce que Spinoza appelle une «notion commune»; et il démontre qu'une telle idée est nécessairement adéquate. Plusieurs cas peuvent alors être considérés suivant qu'une notion commune a sa source dans ce qui est commun à tous les corps ou dans ce qui est commun au corps humain et à certain corps extérieurs, ou encore dans ce qui est commun à deux ou plusieurs hommes (qui se tiennent lieu de corps extérieurs les uns aux autres). Dans le premier cas, les idées obtenues sont celles de "ce qui est commun à toute chose". Ce sont les notions communes les plus universelles, qui sont nécessairement des idées adéquates pour tous ${ }^{11}$. Mais, dans la réalité de l'existence, il semble que les hommes doivent commencer en fait par les moins universelles (à supposer qu'ils y arrivent jamais); cas des idées qui seraient adéquates dans l'esprit d'un ou de plusieurs hommes : "Si le corps humain et certains corps extérieurs, par lesquels le corps humain a coutume d'être affecté, ont quelque propriété commune et qui sont pareillement dans la partie de l'un quelconque des corps extérieurs et dans le tout, de cette propriété aussi, dit Spinoza, l'idée sera dans l'âme adéquate ${ }^{12}$ ».

Spinoza dit : "on ne sait pas ce que peut le corps ${ }^{13}$ ». Nul ne le sait d'avance, avant la mise en œuvre d'une stratégie

11. Ibid., proposition 38 et corollaire, p. 111.

12. Ibid., proposition 39, p. 111-112.

Comme l'explique Gilles Deleuze : "Quand nous rencontrons un corps qui convient avec le nôtre nous éprouvons une affection joyeuse, nous sommes induits à former l'idée de ce qui est commun à ce corps et au nôtre. [...] Cette idée seule, cette notion commune est adéquate. Tel est le second moment de la raison; alors et alors seulement, nous comprenons et agissons, nous sommes raisonnables : non pas par l'accumulation des passions joyeuses et tant que passions, mais par un véritable "saut", qui nous met en possession d'une idée adéquate, à la faveur de cette accumulation" (Spinoza et le problème de l'expression, Paris, Éditions de Minuit, p. 261-262).

13. Éthique, III, 2, scolie, op. cit., p. 139. 
concrète; et aucun homme ne le saura jamais s'il n'essaye pas d'aller au bout de "ce qu'il peut». La stratégie du conatus, en tant qu'il est cet effort de l'homme faisant corps d'aller au bout de ce qu'il peut, consistera à toujours tendre vers une plus grande perfection, c'est-à-dire vers plus de réalité ${ }^{14}$. Mais la question encore une fois est de savoir comment.

"Le corps humain, dit Spinoza, peut être affecté en bien des manières qui accroissent ou diminuent sa puissance d'agir ${ }^{15}{ }^{\prime}$. Lorsque l'individu en est la cause adéquate, les affections s'appellent des actions; dans le cas contraire, ce sont des passions ${ }^{16}$. Une cause est dite adéquate quand l'effet peut en être connu clairement et distinctement par elle seule, sinon elle est dite inadéquate ou partielle" ${ }^{17}$. "Nous sommes actifs, explique Spinoza, quand, en nous ou hors de nous, quelque chose se fait dont nous sommes la cause adéquate»; en revanche, "nous sommes passifs quand il se fait en nous quelque chose ou qu'il suit de notre nature quelque chose, dont nous ne sommes la cause que partiellement ${ }^{18}$ ". Or, les individus sont nécessairement actifs en certaines choses dans la mesure où ils ont des idées adéquates $^{19}$. Car les idées ont une réalité, ou une force ${ }^{20}$, et «rien n'existe, dit Spinoza, de la nature de quoi ne suive quelque effet ${ }^{21}$.

Les notions communes sont les premières idées adéquates que l'individu forme en tant qu'il a quelque propriété commune avec d'autres corps. Or, le pouvoir d'être affecté correspond à certaines propriétés du corps: "Ce qui dispose le corps humain de façon qu'il puisse être affecté d'un plus grand nombre de manières ou le rend apte à affecter les corps extérieurs d'un plus grand nombre de manières, est utile à l'homme; et d'autant plus utile que le corps est rendu par là plus apte à être affecté

14. Ibid., II, définition 6, p. 70.

15. Ibid., III, premier postulat, p. 135.

16. Ibid., définition 3, p. 135.

17. Ibid., définition 1, p. 134.

18. Ibid., définition 2, p. 134.

19. Ibid., proposition 1, p. 135.

20. Cf. Ibid., II, 13, scolie, p. 84.

21. Ibid., I, 36, p. 60. 
et à affecter d'autres corps de plusieurs manières; est nuisible au contraire ce qui diminue cette aptitude du corps ${ }^{22}$ ». Cette croissance, ou passage de l'utile au plus utile, est elle-même une affection, qui constitue une sorte d'instrument de mesure ou d'évaluation de la stratégie, et que Spinoza appelle la joie, passage "d'une moindre à une plus grande perfection ${ }^{23}$ ". L'affection contraire s'appelle la tristesse ${ }^{24}$.

Il est donc nécessaire d'envisager la stratégie du conatus selon deux aspects. Premièrement, à l'intérieur des passions, en faisant jouer l'opposition de la joie et de la tristesse, il s'agit de passer des affections tristes aux affections joyeuses (qui demeurent cependant des passions). Deuxièmement, en faisant jouer cette fois l'opposition de l'être-passif et de l'être-actif, il s'agit de passer de la joie passive à la joie active, c'est-à-dire à la béatitude, autre nom de la liberté. Or, dit Spinoza, "le mieux que nous puissions faire, tant que nous n'avons pas une connaissance parfaite de nos affections, est de concevoir une conduite droite de la vie, autrement dit des principes assurés de conduite, de les imprimer en notre mémoire et de les appliquer sans cesse aux choses particulières qui se rencontrent fréquemment dans la vie ${ }^{25}$ ".

La stratégie de fermeté, c'est-à-dire la stratégie du conatus, qui est pour Spinoza la tâche proprement éthique, est donc liée chez lui à une certaine pratique de la philosophie. Et cette pratique, qui consiste d'abord à envisager la question éthique sous l'angle de la fermeté, en tant qu'elle est un effort pour expliquer les effets par des causes adéquates, vise à l'univocité des termes du langage. Et c'est pourquoi, chez Spinoza, la philosophie commence avec une critique de l'imagination ${ }^{26}$.

La prophétie, par exemple, est liée à l'imagination du prophète; elle n'enveloppe pas de sa nature la certitude. C'est 
pourquoi l'élément du «signe», qui vient confirmer la révélation communiquée parDieuà l'imagination du prophète, doit s'ajouter à la prophétie. "Les prophètes, dit Spinoza, ont toujours eu quelque signe leur donnant la certitude des choses qu'ils imaginaient par le don prophétique ${ }^{27}$ ». Or, Spinoza considère que la connaissance prophétique est à cet égard inférieure à la connaissance naturelle (c'est-à-dire la connaissance parnotions communes), qui n'a besoin d'aucun signe mais enveloppe de sa nature la certitude. La "certitude prophétique, dit-il en effet, n'était pas une certitude mathématique, mais une certitude morale».

Le signe est caractérisé par une ambiguïté essentielle dans la mesure où la prophétie à laquelle il se rattache peut toujours être vraie ou fausse; il est par conséquent objet d'une double lecture. L'interprétation qui pourraitêtre qualifiée de théologique, car elle implique la foi, serait susceptible de lever cette ambiguitté et de conclure en un sens positif (dans le cas de la prophétie authentique). Comme le dit Spinoza : «Une prophétie ou révélation est la connaissance certaine, révélée aux hommes par Dieu, d'une chose quelconque. Quant au prophète, c'est celui qui interprète les choses révélées par Dieu à d'autres personnes incapables d'en avoir une connaissance certaine et ne pouvant donc les saisir que par la foi 28 ".

La position de Spinoza à l'égard de la prophétie est en fait assez paradoxale dans la mesure où il s'adresse vraiment à un "lecteur philosophe»; paradoxale comme la séparation de la foi et de la philosophie, qui est «le but principal» auquel tend tout le Tractatus ${ }^{29}$. Le paradoxe du «lecteur philosophe» consiste à maintenir le signe. Il considère le signe à déchiffrer comme un "hiéroglyphe», à la fois sacré et gravé, selon l'expression même que Spinoza utilise dans le commentaire qu'il fait d'un passage du livre des Nombres (XII, 6) : «Si quelqu'un de vous est un prophète de Dieu, je me révélerai à lui dans une vision". 
Glose de Spinoza : “C'est-à-dire par des figures visibles et des signes à déchiffrer hieroglyphica car, pour la prophétie de Moïse, [le texte de l'Écriture] dit qu'elle est une vision sans obscurité visionem sine hieroglyphicis."

La question de l'interprétation des textes et de l'autorité de l'Écriture est abordée par Spinoza au chapitre VII du Tractatus. II commence ce chapitre en rappelant que «l'Écriture traite très souvent de choses qui ne peuvent être déduites des principes connus par la lumière naturelle». Ce sont les "histoires» et les révélations. Le contenu des premières consiste principalement en "miracles», c'est-à-dire en "récits de faits insolites de la nature adaptés aux opinions et aux jugements des historiens qui les ont écrits ${ }^{30}$ ". Ceux-ci, loin de pouvoir en être déduits, contredisent formellement les principes de la lumière naturelle. En revanche, «les révélations, elles, sont adaptées aux opinions des prophètes, dit Spinoza, de sorte qu'elles dépassent réellement, comme nous l'avons montré au chapitre II [du Tractatus], la compréhension humaine". Et c'est pourquoi elles ne peuvent être déduites des principes connus de la lumière

30. Op. cit., chap. VII, p. 139.

Spinoza remarque aussi par ailleurs que : "Le Psalmiste appelle les miracles d'Égypte des puissances de Dieu parce que, dans une situation d'extrême péril, ils ouvraient une voie de salut” (ibid., chap I, p. 40).

Il y a miracle et miracle. Comme le dira plus tard Wittgenstein dans sa conférence sur l'éthique : «ll est absurde de dire "la science a prouvé qu'il n'y a pas de miracles". En vérité, l'approche scientifique d'un fait n'est pas l'approche de ce fait comme miracle. En effet vous pouvez bien imaginer n'importe quel fait, il n'est pas en soi miraculeux, au sens absolu de ce terme. Car nous constatons maintenant que nous avons employé le mot "miracle» dans un sens relatif et aussi dans un sens absolu. Et je vais maintenant décrire l'expérience qui consiste à s'étonner de l'existence du monde en disant : c'est l'expérience de voir le monde comme un miracle. Je suis alors tenté de dire que la façon correcte d'exprimer dans le langage le miracle de l'existence du monde, bien que ce ne soit pas une proposition du langage, c'est l'existence du langage lui-même" (Leçons et conversations, suivies de Conférence sur l'éthique, Paris, Gallimard, 1971, p. 153).

Et Wittgenstein conclura : «Tout ce à quoi je tendais - et, je crois, ce à quoi tendent tous les hommes qui ont une fois essayé d'écrire ou de parler sur l'éthique ou la religion - c'était d'affronter les bornes du langage" (ibid., p. 155). 
naturelle. La connaissance des révélations de l'Écriture ne peut donc être tirée que de l'Écriture elle-même, comme la connaissance de la nature, de la nature elle-même. Or l'Écriture, pas plus que la nature, ne donne de définitions des "choses" qu'elle contient : definitiones non tradit.

Les définitions que ne donne pas l'Écriture devront être tirées des divers récits, comme celles des choses naturelles sont tirées des diverses actions de la nature ${ }^{31}$. C'est donc la cohérence du texte qui importe, comme le montre le parallèle entre scriptura et natura. Chez Spinoza, le "signe» fait système avec les autres "choses» de l'Écriture, en tant que celle-ci forme elle-même un tout (comme la nature). Maintenir le signe, comme doit le faire celui qui considère l'Écriture en "historien", c'est, comme le dit Spinoza, s'occuper du sens des textes et non de leurvérité; c'est ne pas confondre le sens d'un discours verum sensum avec la vérité des choses rerum veritate. À condition de toujours se situer dans l'Écriture, conclut Spinoza, «quand bien même le sens littéral est en contradiction avec la lumière naturelle, s'il ne s'oppose pas nettement aux principes et aux données fondamentales tirées de l'histoire critique de l'Écriture, il faut le maintenir; au contraire, si ces paroles se trouvaient par leur interprétation littérale contredire aux principes tirés de l'Écriture, encore bien qu'elles s'accordassent le mieux du monde avec la raison, il faudrait admettre une autre interprétation $^{32}$ ".

Maintenir l'inscription du signe, c'est, en se situant en deçà de l'interprétation, garder ouverte la possibilité d'une double lecture : sens littéral et sens métaphorique. Le choix ici n'est pas déterminé par des considérations d'ordre philosophique, mais par la foi qui donne l'intelligence des choses qui vont dans le sens du salut. L'interprétation qui s'accorde avec les données fondamentales tirées de l'Écriture est préférable dans la mesure où celle-ci est tout entière ordonnée au salut. Maintenir, c'est rester "en deçà» du choix à faire: la méthode de Spinoza con- 
siste à chercher «seulement ce que les prophètes ont réellement vu et entendu, non ce qu'ils ont voulu signifier ou représenter par leurs images sensibles hieroglyphicis ${ }^{33}$ ".

Spinoza disait dans la Préface du Tractatus : "J'ai acquis l'entière conviction que l'Écriture laisse la raison absolument libre et n'a rien de commun avec la philosophie, mais que l'une et l'autre se maintiennent par une force propre à chacune ${ }^{34}$ ". Elles se maintiennent l'une et l'autre; la foi aussi est absolument libre. Le dispositif de la séparation de la foi et de la philosophie, dont la mise en place est achevée au chapitre $X V$, est donc un dispositif réversible. Nul ne peut plus prétendre absolument, c'est-à-dire en invoquant la certitude mathématique, que sa philosophie n'est pas l'équivalent d'une «religion», qu'elle n'enseigne pas la soumission à une "nature» qui ne serait au fond qu'un effet d'écriture. Dans la mesure où il est impossible de réduire l'extériorité, la foi et la philosophie doivent avoir partie liée pour bannir la crainte et l'assujettissement. La confusion de l'une et l'autre positions signifierait que «le vulgaire qui ignore le plus souvent les démonstrations, ou est incapable de s'y appliquer, devrait ne rien pouvoir admettre au sujet de l'Écriture que surl'autorité ou par le témoignage des hommes philosophant, et il faudrait, par suite, supposer que les philosophes sont infaillibles dans l'interprétation de l'Écriture; ce serait en vérité une nouvelle autorité ecclésiastique, un nouveau sacerdoce ou une sorte de pontificat qui exciterait dans le vulgaire le rire plutôt que la vénération ${ }^{35}$ ".

Dans ces conditions, il n'est pas difficile de comprendre que l'autorité de l'Écriture doive être corrélative d'une lecture libre (et qui rend libre). Et cela doit être aussi vrai de la philosophie (qui s'écrit) : «Entre ceux qui ne séparent pas la philosophie et la théologie, dit Spinoza, il y a discussion sur le point de savoir si l'Écriture doit être la servante de la raison, ou la raison de l'Écriture; c'est-à-dire si le sens de l'Écriture doit se plier à la

33. Ibid., p. 145.

34. Ibid., Préface, p. 25.

35. Ibid., chap. VII, p. 155. 
raison ou la raison se plier à l'Écriture ${ }^{36}$ ". La première manière de voir est réfutée par Spinoza au chapitre VII du Tractatus, qui traite de l'interprétation de l'Écriture et qui commence par un constat d'erreur : "Tout le monde dit bien omnibus in ore que l'Écriture Sainte est la Parole de Dieu et qu'elle enseigne aux hommes la béatitude vraie ou la voie du salut. La conduite des hommes montre tout autre chose verum re ipsa, car le vulgaire ne paraît se soucier de rien moins que de vivre suivant les enseignements de l'Écriture Sainte, et nous voyons que presque tous substituent à la Parole de Dieu leurs propres inventions et s'appliquent uniquement sous le couvert de la religion à obliger les autres à penser comme eux ${ }^{37}$ ". Contre la seconde manière de voir, Spinoza utilise d'abord un argument négatif, critique. À la question: «La religion et la foi ne peuvent-elles se maintenir que si les hommes s'appliquent laborieusement à tout ignorer et donner à la raison un congé définitif? " Spinoza répond qu'en vérité, «si telle est leur croyance, c'est donc crainte quel'Écriture leur inspire plutôt que confiance ${ }^{38}$ ". Crainte, superstition et servitude s'enchaînent et vont dans la direction opposée au salut. En second lieu, un double argument positif. D'une part: "Que faut-il faire en cas que la raison réclame?" Cas de contradiction de l'Écriture avec la raison: l'exemple du Dieu jaloux ${ }^{39}$. D'autre part, si deux passages de l'Écriture sont directement opposés, auquel des deux faut-il attribuer un sens métaphorique metaphoricè explicare? Cas de contradiction de l'Écriture avec elle-même: pour se tirer d'affaire il faudra bien consulter la raison. Par conséquent, conclut Spinoza, «ni la théologie ne doit être la servante de la raison, ni la raison celle de la théologie, mais l'une et l'autre ont leur royaume propre: la raison, comme nous l'avons dit, celui de la vérité et de la sagesse, la théologie, celui de la piété et de l'obéissance ${ }^{40}$ ". 
La théologie, en tant qu'elle explique la Révélation, a tout pouvoir de déterminer les dogmes de la foi dans son domaine propre, qui est celui de l'obéissance, but auquel tend l'Écriture. Pour Spinoza il ne fait aucun doute que la théologie ainsi comprise, et dans les enseignements qu'elle donne pour la vie documenta vitae, ne peut ni ne veut entrer en conflit avec la raison (le salut du croyant comme celui du philosophe est lié à une même exigence : être dans le vrai). Toutefois, la raison ne peut établir la vérité ou la fausseté du principal enseignement de la théologie : le salut parl'obéissance. La liberté du philosophe a comme contrepartie la liberté du croyant; telle semble être du moins la position de Spinoza. Mais cette liberté, chez le croyant, a un prix: quel type de certitude peut avoir en effet celui qui n'est pas philosophe? "Je réponds, dit Spinoza, que j'admets absolument que le dogme fondamental de la théologie ne peut être découvert investigari par la lumière naturelle ou que du moins nul ne s'est trouvé qui l'ait démontré, et par suite que la Révélation a été nécessaire au plus haut point; que néanmoins nous pouvons justifier notre adhésion à ce dogme révélé de façon à avoir à son sujet une certitude au moins morale ${ }^{41}$ ». Une certitude morale à défaut d'une certitude mathématique, qui serait celle de la philosophie, c'est-à-dire celle d'une éthique "démontrée suivant l'ordre géométrique". Donc, en fait, une certitude au plus morale.

L'autorité de l'Écriture, en effet, dépend de l'autorité des prophètes. Or, Spinoza a montré au chapitre II que la certitude des prophètes a été tout au plus morale; car celui qui s'engage dans la voie de l'obéissance ne peut, comme il dit, "prétendre à une certitude d'ordre plus élevé». Comme l'explique clairement, du reste, la note marginale II du chapitre I : «Si les hommes qui écoutent les prophètes devenaient prophètes, comme deviennent philosophes ceux qui écoutent les philosophes, le prophète ne serait pas un interprète des décrets divins, puisque ses auditeurs ne s'appuieraient plus sur le témoignage et l'autorité du prophète lui-même, mais aussi sur une révélation 
et un témoignage interne ${ }^{42}$ ». Croire à l'Écriture, c'est croire aux prophètes. Or, ceux-ci ont enseigné un chemin vers la béatitude par l'obéissance et la foi, et ils ont confirmé leur enseignement par des signes. Mais ces signes n'ont-ils pas été accomplis dans le passé (l'Écriture n'en aurait gardé en somme que la trace)?

II est difficile de se fier à la lettre de l'Écriture, ce papier, cette encre, matière exposée à la corruption ${ }^{43}$. La méfiance naturelle du philosophe ne risque-t-elle pas, dans ces conditions, de réduire le signe à rien? La confirmation la plus sûre, en tous cas, se trouve pour Spinoza dans l'accord entre la raison et la doctrine, en tant que celle-ci donne un enseignement moral : "Tous peuvent obéir, dit-il, et seule une partie comparativement très petite du genre humain atteint l'état de vertu sous la seule conduite de la raison; si donc nous n'avions pas le témoignage de l'Écriture, nous douterions du salut de presque tous ${ }^{44}$ ". Voilà de quelle façon se termine la transition vers la partie proprement politique du traité. Or qu'en est-il, dans ce nouveau contexte, de la question d'autrui et d'une possible mais fort improbable "éthique de la générosité»?

Le développement de la question éthique, envisagée sous l'angle de la fermeté, s'explique à première vue par une stratégie du conatus. Considérée par ce biais, la question éthique n'est pas autre chose que celle du désir comme essence de l'individu qui, une fois entré dans l'existence, va aussi loin qu'il peut : désir dont la limite réside dans le degré de puissance qu'il représente (et qui n'est pas infini), et dans une certaine durée qu'il a (et qui n'est pas non plus indéfinie); désir qui se caractérise par la recherche de l'utile propre et qui, en tant que tel, est appelé fermeté par Spinoza. Le commandement de la raison, en vertu duquel l'individus'efforce de se conserver, le pousseà s'associer aux autres hommes, ce qui ne va pas sans la prise en compte, le cas échéant, de l'intérêt d'autrui. D'où la nécessité de définir la générosité, à quoi pourront être rapportées les actions qui ont

42. Ibid., notes marginales (ajoutées par Spinoza), p. 339.

43. Ibid., chap. XII, p. 220.

44. Ibid., chap. XV, p. 257. 
pour but l'utilité d'autrui et par lesquelles un individu s'efforce en vertu du seul commandement de la raison d'assister les autres hommes et d'établir entre eux et lui un lien d'amitié. Or, pour Spinoza, la nature ne crée pas des nations mais des individus; ceux-ci doivent opérer des regroupements (par une décision qui établit la règle de droit). Nul n'ayant le temps ni les forces qu'il faudrait pour labourer, semer, moissonner, moudre, cuire, tisser et effectuer les autres travaux utiles à l'entretien de la vie et nécessaires à la perfection de sa nature, l'assistance mutuelle est indispensable; et c'est ce qui requiert la formation d'une société. Mais l'entraide, au départ, ne va pas de soi (il faut le passage de la nature à la culture).

L'articulation de la fermeté et de la générosité, qui est sousjacente au rapport entre individu et société (envisagé du point de vue de la raison), pose un problème sitôt qu'apparaît l'éventualité d'un conflit: qu'est-ce qui peut assurer que telle ou telle action, qui doit en tout état de cause avoir pour but l'utilité de l'agent, aura effectivement aussi pour but l'utilité d'autrui (à supposer encore, évidemment, qu'il s'agisse d'un ami et non d'un ennemi)? II y a deux manières d'aborder le problème, suivant Spinoza, chacune indiquant une voie de salut. La première, celle de la philosophie, présuppose des hommes "gouvernés par la raison". Or, il ne peut y avoir de conflit entre hommes raisonnables; la raison ne peut pas être divisée contre elle-même (ce serait absurde). Pour de tels hommes, la recherche de l'utile propre enveloppe la générosité : "ceux qui cherchent ce qui leur est utile sous la conduite de la raison, dit Spinoza, n'apprêtent rien pour eux-mêmes qu'ils ne désirent pour les autres hommes, et sont ainsi justes, de bonne foi et honnêtes." Sous la conduite de la raison, les hommes ne peuvent qu'aboutir à la formation d'un corps social, s'efforçant ensemble de conserver leur être et cherchant ensemblel'utilité commune; car si «deux individus entièrement de même nature se joignent l'un à l'autre, dit Spinoza, ils composent un individu deux fois plus puissant que chacun séparément. Rien donc de plus utile à l'homme que l'homme ${ }^{45}$ ".

45. Éthique, IV, 18, scolie, p. 237. 
La seconde manière acceptable aux yeux de Spinoza est celle de la foi (admise par lui comme voie de salut en vertu du témoignage de l'Écriture); elle met en œuvre le modèle du signe issu de la lecture spinoziste de la Bible. Du point de vue de la philosophie, les signes ont le statut de traces, comme le précise le passage suivant: "Un soldat, par exemple, ayant vu sur le sable les traces d'un cheval, passera aussitôt de la pensée d'un cheval à celle d'un cavalier, et de là à la pensée de la guerre, etc. Un paysan, au contraire, passera de la pensée d'un cheval à celle d'une charrue, d'un champ, etc. ${ }^{46}$ ". L'interprétation des signes est fonction de la diversité des paradigmes; la trace n'est que le lieu de certains choix stratégiques possibles entre des séries. Le "lecteur philosophe" doit s'abstenir de prendre position: ni soldat, ni paysan, etc. (la philosophie ne peut envisager la trace que sur le mode de l'absence). Seule la mémoire permet une interprétation des signes qui les inscrit dans l'expérience vécue du rapport à une présence.

L'autre homme estl'énigme par excellence : autrui montrant son visage, autrui se présentant en son être corporel, réalité sensible de la communication. Un homme rencontre son semblable; cette rencontre est-elle bonne ou mauvaise? Une certaine force d'âme ou fermeté, pense-t-il, pousse l'autre à persévérer dans son être; mais cet individu agit-il en vertu du seul commandement de la raison? Dans la négative, c'est un adversaire potentiel et potentiellement dangereux; la perspective est celle de l'éventualité du conflit. Comme le rappelle Spinoza, «il n'est donné dans la nature aucune chose singulière qu'il n'en soit donnée une autre plus puissante et plus forte [...] par laquelle la première peut être détruite ${ }^{47}$ ». L'attitude appropriée

46. Ibid., II, 18, scolie, p. 97.

47. Ibid., IV, axiome, p. 221.

D'où la question que formulera Max Weber : "Quelle est, indépendamment des buts qui lui sont propres, la mission que la politique peut remplir dans l'économie globale de la conduite de la vie? Quelle est pour ainsi dire le lieu éthique où elle réside?" (Le savant et le politique, Paris, Plon, 1959, p. 181). L'éthique peut parfois comme il le dit, jouer un rôle extrêmement fâcheux : "Une nation pardonne toujours les préjudices matériels qu'on lui fait subir, mais non une atteinte portée à son honneur, surtout lorsqu'on emploie la manière d'un prédicateur qui veut avoir raison à tout prix. Tout nouveau document porté à la connaissance publique des dizaines 
est à première vue la méfiance. La générosité, en revanche, à condition d'être perçue et reçue comme telle rendrait possible une forme de confiance; elle ouvrirait un espace de communication. Dans la rencontre véritable, le corps d'autrui fait signe (comme les signes de l'Écriture forment un corpus). L'attitude corrélative est ici celle de la foi en l'autre comme interlocuteur (capable de donner sa parole).

Ainsi donc en est-il de la rencontre d'autrui comme du témoignage de l'Écriture, qui renvoie à l'autorité du prophète ${ }^{48}$. Les deux renvoient à la question même de l'autorité. Qu'est-ce qui donne confiance, ou qu'est-ce qui fait vivre? La parole, une parole humaine, a-t-elle ce pouvoir? L'individu a-t-il quelque chose à gagner à ce jeu du langage? Peut-il même nommer ce qui se trouve yêtre l'objet de son désir? «Pour la joie née du bien d'autrui, dit Spinoza, je ne sais de quel nom il faut l'appeler ${ }^{49}$ ".

d'années après ne peut avoir d'autre résultat que de réveiller des clameurs indignes, la colère et la haine, alors qu'il vaudrait mieux enterrer la guerre, moralement au moins, lorsqu'elle est terminée. Mais une telle attitude n'est possible que si l'on a le sens des réalités, le sens chevaleresque, et avant tout le sens de la dignité. Elle se défend donc d'afficher une "éthique" qui, en vérité, témoigne toujours d'une absence de dignité des deux cótés. Ce dernier genre d'éthique ne se préoccupe jamais que de la culpabilité dans le passé, question stérile du point de vue politique parce qu'insoluble, au lieu de s'intéresser à l'affaire propre de l'homme politique, à savoir l'avenir et la responsabilité devant l'avenir. S'il existe des crimes politiques, cette façon de procéder en est un. Par ailleurs, une telle attitude a l'inconvénient supplémentaire de nous cacher à quel point tout le problème est faussé par les intérêts matériels : intérêts du vainqueur à tirer le plus grand profit possible de sa victoire - qu'il s'agisse d'intérêts matériels ou moraux -, espoirs du vaincu de marchander l'aveu de sa culpabilité contre certains avantages. S'il existe au monde quelque chose d' "abject", c'est bien cela. Voilà ce qu'il en résulte lorsqu'on veut utiliser l'éthique pour avoir toujours raison" (Ibid., p. 182-183).

48. Le prophète est aussi un auteur. Et le salut vient dans ce cas de l'autorité de l'auteur, du latin auctor, "qui fait profiter" ou "qui fait grandir".

49. Éthique, III, 22, scolie, op. cit., p. 156.

Or, «la vraie félicité et la béatitude, dit Spinoza, ne consistent pour chacun que dans la jouissance du bien et non dans cette gloire d'être le seul à en jouir, les autres étant exclus; s'estimer en possession d'une béatitude plus grande, en effet, parce qu'on est seul dans une condition bonne, les autres non, ou parce qu'on jouit d'une béatitude plus grande et qu'on a meilleure fortune que les autres, c'est ignorer la vraie félicité et la béatitude; la joie qu'on éprouve à se croire supérieur, si elle n'est pas toute enfantine, ne peut naître que de l'envie et d'un mauvais cœur" (Traité théologico-politique, chap. III, op. cit., p. 69). 
Comment savoir si l'éthique n'est pas un stratagème? En amour comme à la guerre, toute ruse est bonne $e^{50}$. La question de la vérité de l'éthique, comme le montre la problématique particulière du signe dans la philosophie de Spinoza, est inéluctable. II ne fait aucun doute que l'existence de ce genre de question est caractéristique du domaine de l'éthique; mais non moins caractéristique est l'absence de solution. Cela est indécidable.

Pierre Gendron

Centre de recherche en droit public Université de Montréal

50. La ruse, ici, ne signifie pas autre chose que la supériorité de l'esprit de finesse sur l'esprit de géométrie. Comme l'explique François Marty : "La communication est, de soi, fort simple : il suffit de dire à son interlocuteur ce que l'on veut lui dire. Cela suppose, il est vrai, un préalable, tellement important qu'il doit accompagner tout au long l'entreprise, celui de la foi mutuelle, qui croit - le terme est très précis - en l'intérêt de la communication et en sa réussite possible. C'est même cette foi mutuelle qui va conduire à la plus grande ingéniosité quand la communication se manifeste moins simple qu'il n'y paraissait d'abord : répétitions, variantes diverses, gestes, allusions aux objets de l'environnement, tout est bon pour la faire réussir" (La bénédiction de Babel, Paris, Cerf, 1990, p. 77-78). 